\title{
Interleukin-1beta Causes Fluoxetine Resistance in an Animal Model of Epilepsy-Associated Depression
}

\author{
Eduardo A. Pineda • Julie G. Hensler • Raman Sankar • \\ Don Shin • Teresa F. Burke • Andréy M. Mazarati
}

Published online: 17 March 2012

(C) The American Society for Experimental NeuroTherapeutics, Inc. 2012

\begin{abstract}
Depression represents a common comorbidity of epilepsy and is frequently resistant to selective serotonin reuptake inhibitors (SSRI). We tested the hypothesis that the SSRI resistance in epilepsy associated depression may be a result of a pathologically enhanced interleukin- $1 \beta$ (IL1- $\beta$ ) signaling, and consequently that the blockade of IL1- $\beta$ may restore the effectiveness of SSRI. Epilepsy and concurrent depression-like impairments were induced in Wistar rats by pilocarpine status epilepticus (SE). The effects of the 2-week long treatment with fluoxetine, interleukin-1 receptor antagonist (IL-1ra), and their combination were examined using behavioral, biochemical, neuroendocrine, and autoradiographic assays. In post-SE rats, depression-like impairments included behavioral deficits indicative of hopelessness and anhedonia; the hyperactivity of the hypothalamo-pituitaryadrenocortical axis; the diminished serotonin output from raphe nucleus; and the upregulation of presynaptic serotonin 1-A (5-HT1A) receptors. Fluoxetine monotherapy exerted no antidepressant effects, whereas the treatment with IL-1ra led to the complete reversal of anhedonia and to a partial improvement of all other depressive impairments. Combined administration of fluoxetine and IL-1ra completely abolished all hallmarks of epilepsy-associated depressive abnormalities, with the exception of the hyperactivity of the hypothalamopituitary-adrenocortical axis, the latter remaining only partially
\end{abstract}

E. A. Pineda $\cdot$ R. Sankar $\cdot$ D. Shin $\cdot$ A. M. Mazarati $(\bowtie)$ Department of Pediatrics, Neurology Division, David Geffen

School of Medicine, University of California Los Angeles,

Los Angeles, CA 90095, USA

e-mail:mazarati@ucla.edu

J. G. Hensler • T. F. Burke

Department of Pharmacology,

University of Texas Health Science Center-San Antonio,

San Antonio, TX 78229, USA improved. We propose that in certain forms of depression, including but not limited to depression associated with epilepsy, the resistance to SSRI may be driven by the pathologically enhanced interleukin- $1 \beta$ signaling and by the subsequent upregulation of presynaptic 5-HT1A receptors. In such forms of depression, the use of interleukin-1 $\beta$ blockers in conjunction with SSRI may represent an effective therapeutic approach.

Keywords Epilepsy - depression · comorbidity · cytokines · selective serotonin reuptake inhibitors

\section{Introduction}

Status epilepticus (SE) induced in rats by lithium chloride $(\mathrm{LiCl})$ and pilocarpine, along with spontaneous recurrent seizures, leads to behavioral changes indicative of depression [1]. The state mimicking hopelessness and despair stems from the diminished release of serotonin (5-HT) from raphe nuclei into the terminals [2]. In turn, the compromised serotonergic transmission is due to the functional upregulation of presynaptic serotonin type 1A (5-HT1A) receptors [3], which normally control 5-HT release from raphe nuclei on a negative feedback basis [4-6]. Finally, the upregulation of 5-HT1A autoreceptors is driven by the dysregulation of the hypothalamo-pituitaryadrenocortical (HPA) axis [1, 7], which (dysregulation) by itself represents a neuroendocrine hallmark of chronic stress [8]. At the same time, behavioral impairment indicative of anhedonia has not been associated with the deficit of serotonergic transmission [2].

On the basis of the reported findings, we have suggested that chronic sequelae of pilocarpine SE can be regarded as a model of comorbidity between epilepsy and depression [1], 
which represents 1 of the most frequent neuropsychiatric comorbidities of epilepsy [9]. Concrete mechanisms of epilepsy-associated depression remain by and large elusive, although some studies have implicated the dysregulation of the HPA axis [10], and the upregulation of presynaptic 5-HT1A receptors [11].

Our experimental therapy studies established that all depression-like impairments: i) persisted despite effective suppression of spontaneous seizures by topiramate (personal unpublished data); ii) were resistant to the treatment with a selective serotonin reuptake inhibitor (SSRI) selective 5-HT reuptake inhibitor fluoxetine [2]; and iii) could be either partially improved (HPA axis dysregulation, diminished serotonergic transmission and despair) or completely reversed (anhedonia) by interleukin-1 receptor antagonist (IL-1ra) [12], a compound that selectively antagonizes a cytokine interleukin-1 $\beta$ (IL-1 $\beta$ ) at the level of its receptor, IL1-RI $[13,14]$ (the effects of IL-1ra on the function of 5-HT1A receptors have not been explored yet). The effects if IL-1ra led us to propose that the increased IL- $1 \beta$ signaling, which has been a well-established hallmark of temporal lobe epilepsy $[15,16]$, may also be the upstream-most factor that via the hyperactivity of the HPA axis [17-20] leads to the development of depressive impairments [1]. It has been long suggested that epilepsy and depression share certain pathophysiological mechanisms $[21,22]$. Given the involvement of IL-1 $\beta$ in epilepsy on the one hand and in depression on the other hand $[17,23]$, it is conceivable to suggest that this cytokine represents one of factors linking mechanisms of the 2 diseases.

The resistance to fluoxetine and the inability of IL-1ra to completely abolish depressive impairments in epileptic animals have induced us to further explore therapeutic strategies for epilepsy-associated depression. SSRI resistance represents a serious challenge in the treatment of major depressive disorder, with therapeutic response lacking in 30 to $50 \%$ of patients $[24,25]$. The situation is also true for epilepsy-associated depression, whereby SSRI are effective in 60 to $70 \%$ of patients [26-28], thus leaving a significant fraction of the population in the need of alternative antidepressant therapies.

In the present study, using an experimental therapy approach, we explore the possibility that the resistance to SSRI may stem from the pathologically enhanced brain IL- $1 \beta$ signaling, which is in itself triggered by the epileptic process.

\section{Methods}

\section{Experimental Subjects}

The experiments were performed in male Wistar rats (Charles River, Wilmington, MA), 50 days old at the beginning of the study, in accordance with the policies of the National Institutes of Health.
Status Epilepticus and Spontaneous Seizures

SE was induced by $\mathrm{LiCl}(128 \mathrm{mg} / \mathrm{kg}$, i.p.) followed by pilocarpine $(40 \mathrm{mg} / \mathrm{kg}$, s.c.) $24 \mathrm{~h}$ later. After seizure onset (at 3 and $8 \mathrm{~h})$, rats were injected with diazepam $(10 \mathrm{mg} / \mathrm{kg}$, i.p. $)$ and phenytoin $(50 \mathrm{mg} / \mathrm{kg}$, i.p.) $[2,3,7]$. In control animals (further defined as naïve), pilocarpine was substituted with saline. All experiments started 2 months after SE. To confirm the development of chronic epilepsy, animals were subjected to continuous 2 or more weeks of video monitoring for the detection of stage 4 to 5 seizures (i.e., rearing and/or rearing and falling). To avoid immediate effects of spontaneous seizures on outcome measurements, all assays were performed on verification that no seizures had developed for at least 6 hours prior to or during the test $[2,3,7]$.

\section{Forced Swim Test}

Animals were placed individually inside of a plastic nontransparent tank (height $60 \mathrm{~cm}$, diameter $45 \mathrm{~cm}$ ) for 5 minutes. Active swimming (attempts to escape) and immobility (movements purposed only to maintain the head above the water) were recorded. The increased immobility time has been regarded as an equivalent of hopelessness and despair [2, 3, 7].

\section{Taste Preference Test}

Two identical bottles were placed in the animal's cage: 1 filled with $0.1 \%$ saccharin and another with regular water. Taste preference was expressed as percentage of saccharin solution of a total fluid (saccharin plus water) consumed within $24 \mathrm{~h}$. The consumption of statistically equal volumes of saccharin and water is interpreted as an equivalent of the state of anhedonia [2, 12].

\section{Combined Dexamethasone/Corticotropin-Releasing Hormone Test}

Blood (50 $\mu$ l, i.v.) from the blood that was collected from the tail vein, and dexamethasone (DEX) $(30 \mu \mathrm{g} / \mathrm{kg}$, i.v.) were injected. A second blood sample was collected $6 \mathrm{~h}$ after DEX injection, followed by the injection of corticotrophinreleasing hormone (CRH) $(50 \mathrm{mg} / \mathrm{kg}$, i.v. $)$. A third blood sample was collected 30 minutes after the CRH injection. Corticosterone (CORT) was measured using radioimmunoassay (Immunochem Double Antibody Corticosterone ${ }^{125} \mathrm{I}$ radioimmunoassay kit, MP Biomedicals, Orangeburg, NY) in $10 \mu \mathrm{l}$ plasma samples. The dysregulation of the HPA axis consists of the failure of DEX to suppress plasma CORT and of the exacerbated increase of plasma CORT in response to CRH $[3,7,12]$. 
Fast Cyclic Voltammetry

Under the urethane anesthesia, animals were implanted with a bipolar stimulating electrode into dorsal raphe (Bregma -7.8 $\mathrm{mm}$; midline; ventral $6.5 \mathrm{~mm}$ ) and with a Nafion-coated carbon fiber electrode (World Precision Instruments, Sarasota, FL) into the sensorimotor cortex. The release of 5-HT in the cortex was induced by electrical stimulation of raphe (bipolar square wave pulses, $100 \mathrm{~Hz}, 200 \mathrm{~ms}, 0.35 \mathrm{~mA}$ ). The amount of released 5-HT was measured by applying the ramp current to the carbon fiber electrode (scanned from $0.2 \mathrm{~V}$ to $1 \mathrm{~V},-0.1$ $\mathrm{V}$ and $0.2 \mathrm{~V}$, at $1000 \mathrm{~V} / \mathrm{s}$ ) and the acquisition of oxidative peaks using POT500 scanning potentiostat (World Precision Instruments) before and after raphe stimulation [2, 3]. The difference between the 2 peaks (referred to as faradaic current) reflects the oxidation of 5-HT into quinone and reflects the amount of transmitter released in response to raphe stimulation [29].

\section{5-HT1A Receptor Autoradiography}

The animals were decapitated and the brains were removed and stored at $-80^{\circ} \mathrm{C}$. Coronal $20-\mu \mathrm{m}$ thick sections were cut at the level of dorsal raphe (Bregma -7.64 to $-8.00 \mathrm{~mm}$ ). The functional capacity of 5-HT1A receptors to activate G-protein was examined using $\left[{ }^{35} \mathrm{~S}\right] \mathrm{GTP} \gamma \mathrm{S}$ autoradiography. Sections were incubated with $40 \mathrm{pmol} / \mathrm{L}\left[{ }^{35} \mathrm{~S}\right] \mathrm{GTP} \gamma \mathrm{S}$, either in the absence or in the presence of the 5-HT1A receptor agonist $( \pm)$-8-hydroxy2-dipropylaminotetralin (8-OH-DPAT) at concentrations of 15 nmol or $1 \mu \mathrm{mol}$, which produce 50 and $100 \%$ responses, respectively. Nonspecific $\left[{ }^{35} \mathrm{~S}\right] \mathrm{GTP} \gamma \mathrm{S}$ binding was defined in the absence of 8-OH-DPAT and in the presence of $10 \mu \mathrm{mol} / \mathrm{L}$ GTP $\gamma$ S. Sections were exposed to Kodak Biomax MR film (Amersham, Piscataway, New Jersey) for 48 h. Digitized autoradiograms were analyzed using NIH Image software (National Institutes of Health, Bethesda, MD, ImageJ 1.42q) [3, 30].

\section{Treatments}

Human recombinant interleukin-1 receptor antagonist (IL-1ra; Kineret, Biovitrum, Sweden) was administered (intracerebroventricularly) into the lateral brain ventricle continuously for 2 weeks. Under isoflurane anesthesia, animals were implanted intracerebroventricularly with a 28-gauge cannula connected via a polypropylene catheter to the ALZET osmotic mini-pump model 2002 (total volume, $200 \mu$ l, delivery rate $0.5 \mu \mathrm{l} / \mathrm{h}$, duration 2 weeks; Durect Corp, Cupertino, CA), with the latter placed subcutaneously on the back. The pump contained IL-1ra diluted in normal saline. Fluoxetine (Spectrum Chemical, Gardena, CA) was injected intraperitoneally once a day, for 2 weeks. Control animals received both vehicles simultaneously.
Test Sequence

Forced swim test (FST) and taste preference tests were performed on days 10 and 11 after the start of the treatments. The DEX/CRH test was performed on day 12 and/or day 13 . On day 14 , the animals were processed for either 5-HT1A autoradiography or for fast cyclic voltammetry.

Sample sizes and statistical methods are indicated in the figure legends.

\section{Results}

\section{Dose Selection}

In pilot experiments, doses of fluoxetine and IL-1ra were chosen based on the effects of the compounds on behavior of post-SE animals in the FST. IL1-ra shortened the immobility time when administered at 200, 500, and $1000 \mu \mathrm{g}$ for 2 weeks (Fig. 1). Animals treated with IL-1ra at $1500 \mu \mathrm{g}$, exhibited weight loss of $\geq 10 \%$, lack of grooming, periorbital bleeding, and significantly worsened performance in the FST (Fig. 1a). For further studies, the $500 \mu \mathrm{g}$ dose of IL-1 ra was chosen. Fluoxetine had no effect on the behavior of the animals at doses of $10 \mathrm{mg} / \mathrm{kg}$ (Fig. 2) and $20 \mathrm{mg} / \mathrm{kg}$ (not shown). At $20 \mathrm{mg} / \mathrm{kg}$, animals exhibited significant weight loss $(\geq 10 \%)$. For further studies, the $10 \mathrm{mg} / \mathrm{kg}$ dose was selected.

\section{Behavioral Equivalents of Depression}

Post-SE animals showed a significant increase in the immobility time in the FST, and the absence of preferential saccharin consumption, which suggested the development of hopelessness-like and anhedonia-like impairments, respectively [2] (Fig. 2a, b, control vs naïve). IL-1ra significantly improved behavior in the FST (IL-1ra vs control), although the performance of the animals was still significantly worse than in the naïve rats (IL-1ra vs naïve). Furthermore, IL-1ra completely restored saccharin preference in epileptic rats. Fluoxetine had no effect on either of the behavioral deficits. Combined administration of IL-1 ra and fluoxetine brought the immobility time in the FST down to levels observed in naïve animals (Fig. 2a, IL-1ra + fluoxetine [FLX] vs naïve).

\section{Dysregulation of the HPA Axis}

In post-SE rats, the dysregulation of the HPA axis was evident, as the positive DEX/CRH test (i.e., the failure of DEX to decrease plasma CORT level) and the exacerbated rise of plasma CORT in response to $\mathrm{CRH}$ injection [7] (Fig. 3, control vs naïve). IL-1ra administration yielded the responsiveness to DEX and mitigated $\mathrm{CRH}$-induced increase 

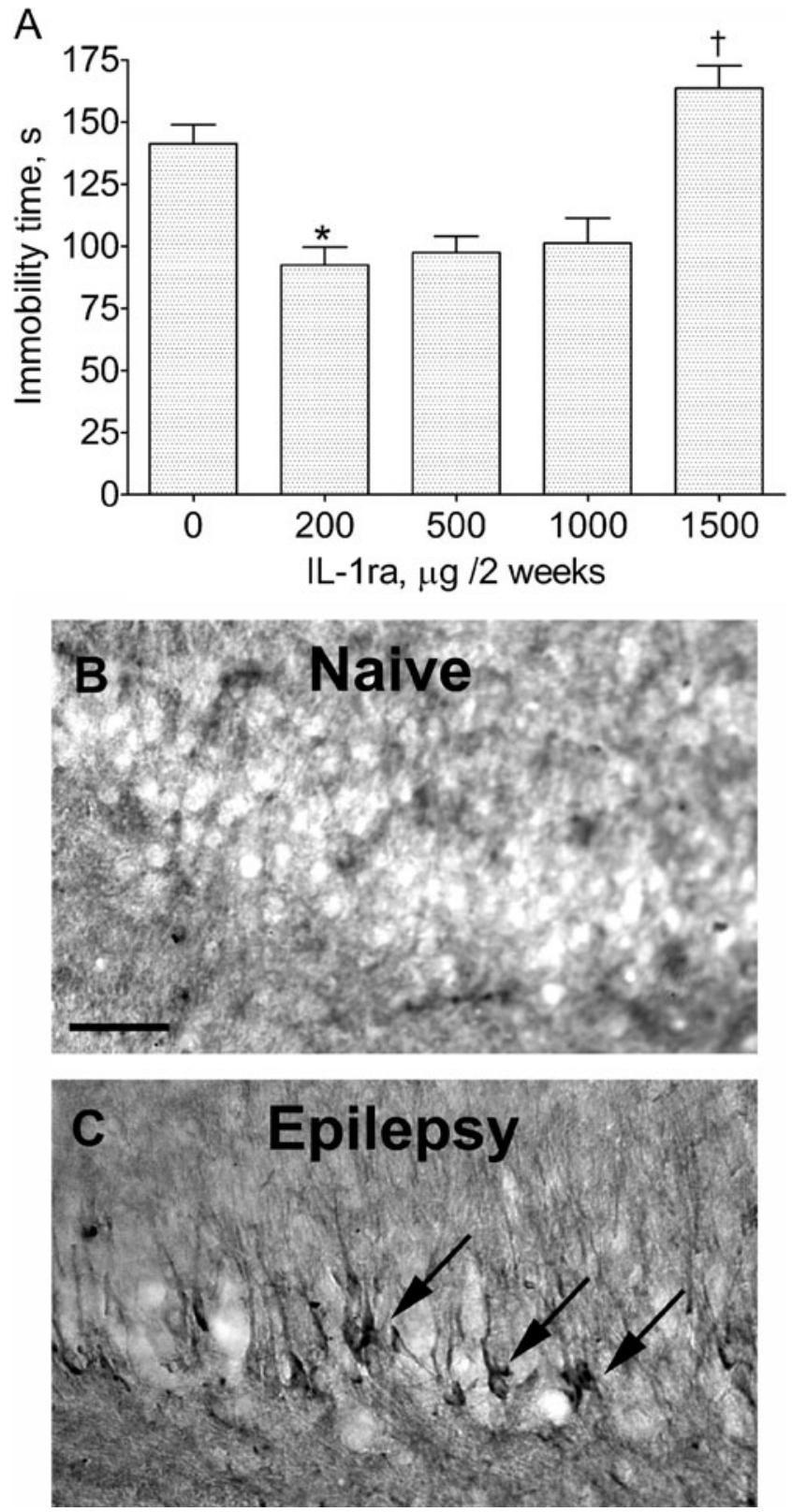

Fig. 1 (a) Effects of various doses of interleukin-1 receptor antagonist (IL-1ra) in post-status epilepticus (SE) rats on the immobility time in the forced swim test (FST). The effects of 200, 500, and $1000 \mu \mathrm{g}$ were statistically similar. At $1500 \mu \mathrm{g}$ IL-1ra treatment worsened the performance of the animals in the FST. Each group included 4 animals. Data are shown as mean \pm SEM. * $p<0.05 v s$ " $0 \mu \mathrm{g}$ "; ${ }^{\dagger} p<0.05 v s$ "200 $\mu \mathrm{g}$ " (Kruskal-Wallis test followed by Mann-Whitney $U$ post hoc test). $(\mathbf{b}, \mathbf{c})$ Immunohistochemical staining of interleukin-1 $\beta$ (IL-1 $\beta$ ) in the CA3 area of the hippocampus of a naïve rat (b) and a rat 2 months after SE and documented spontaneous seizures (c). Note the appearance of IL-1 $\beta$-positive cells (indicated by arrows) in the post-SE rat. Scale bar $=40 \mu \mathrm{M}$

of plasma CORT level (IL-1 ra vs control), although both values were still outside of normal parameters (IL-1ra $v s$ naïve). Fluoxetine treatment did not modify the reactivity of the HPA axis. Combined treatment with IL-1ra and fluoxetine did not
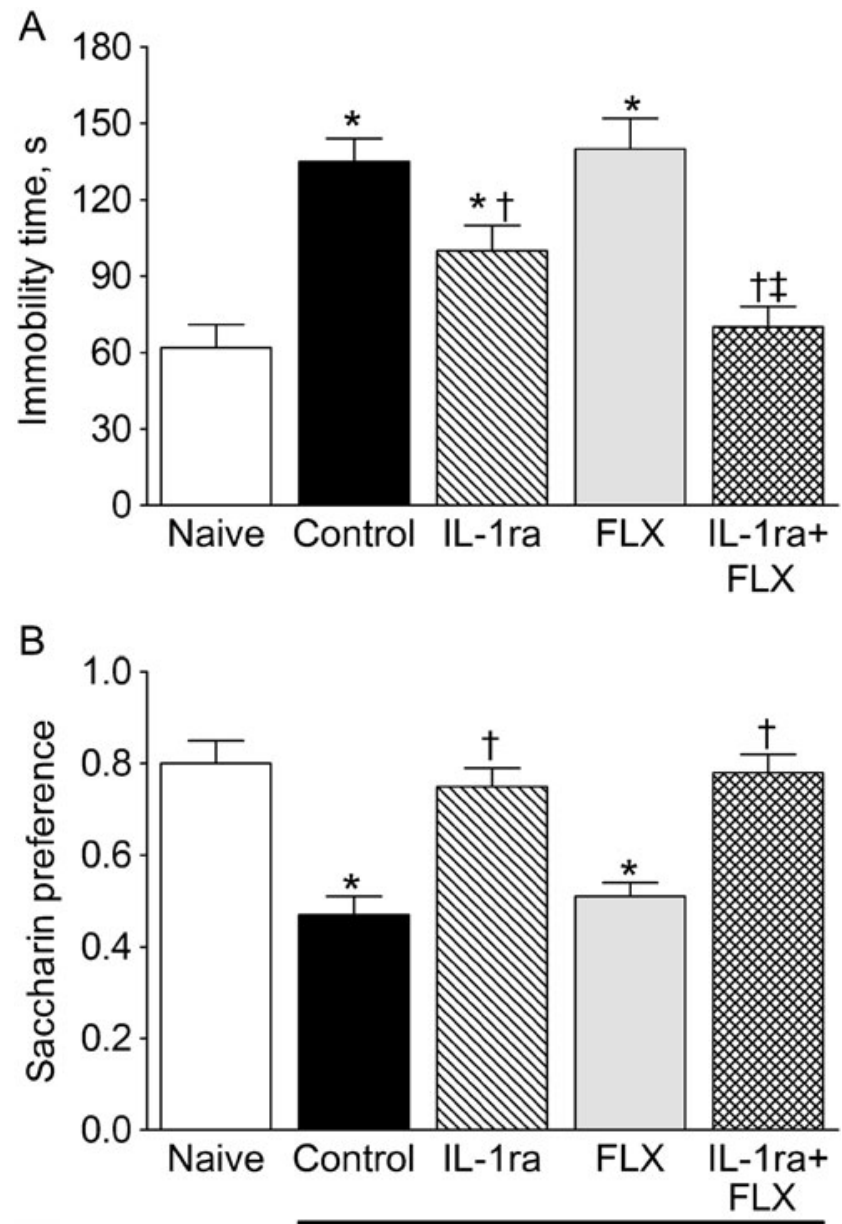

Post-SE

Fig. 2 Effects of interleukin-1 receptor antagonist (IL-1ra), fluoxetine (FLX), and their combination on behavioral depressive impairments in post-status epilepticus (SE) rats. (a) Effects on the immobility time in the forced swim test (FST). (b) Effects on the saccharin versus water consumption in the taste preference test. Each group included 12 animals. Data are shown as mean \pm SEM. ${ }^{*} p<0.05$ versus naïve; ${ }^{\dagger} p<0.05$ versus SE alone; ${ }^{\star} p<0.05$ versus IL-1ra alone and versus FLX alone (one-way analysis of variance+Neuman-Keuls post hoc test)

result in further improvement of the HPA axis response, as compared with the IL1-ra monotherapy (Fig. 3).

\section{Evoked 5-HT Release}

In post-SE animals, electrical stimulation of raphe nucleus induced significantly smaller 5-HT oxidative currents in the cortex than in naïve rats (Fig. 4), thus pointing toward the diminished serotonergic tone [2]. In IL-1ra treated post-SE rats, the evoked 5-HT release was in between and statistically similar to the values detected for naïve and untreated post-SE rats (Fig. 4). No improvement in 5-HT release was observed with fluoxetine treatment. The combined administration of IL$1 \mathrm{ra}$ and fluoxetine led to a significant increase of 5-HT release, which exceeded the level detected in naïve subjects (Fig. 4a, b, IL-1ra + FLX vs naïve). 


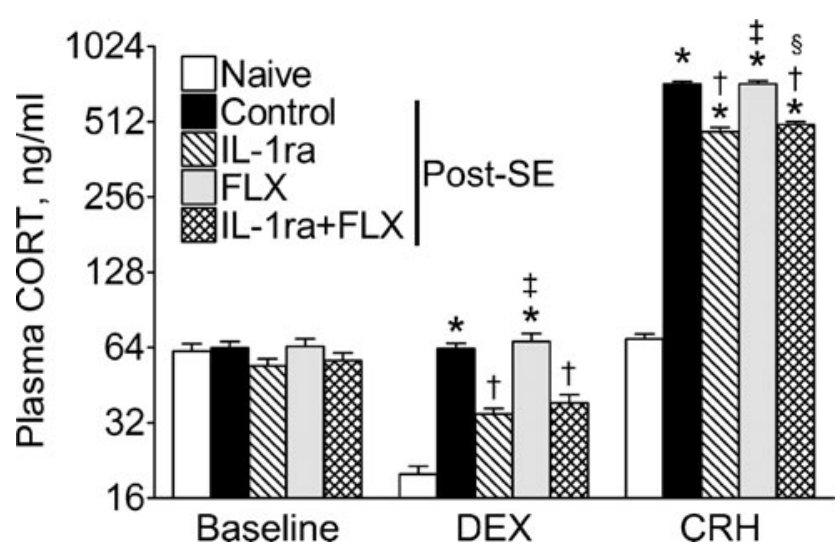

Fig. 3 Effects of interleukin-1 receptor antagonist (IL-1ra), fluoxetine (FLX), and their combination on the outcome of the combined dexamethasone (DEX)/corticotrophin-releasing hormone (CRH) test. Sample sizes: naïve $\mathrm{n}=9$; control $\mathrm{n}=12$; IL-1 ra $\mathrm{n}=9$; FLX $\mathrm{n}=8$; IL-1 ra + FLX $\mathrm{n}=8$. Data are shown as mean \pm SEM. ${ }^{*} p<0.05$ versus naïve; ${ }^{\dagger} p<0.05$ versus $\mathrm{SE}$ alone; ${ }^{*} p<0.05$ versus IL-1 ra alone; ${ }^{\S} p<0.05$ versus FLX alone (two-way repeated measures analysis of variance + Bonferroni post hoc test). DEX significantly suppressed plasma CORT only in naïve animals, and CRH significantly increased plasma corticosterone (CORT) in all groups (oneway repeated measures analysis of variance + Bonferroni post hoc test). $\mathrm{SE}=$ status epilepticus

\section{Raphe 5-HT1A Receptor Function}

Functional upregulation of presynaptic 5-HT1A receptors in post-SE animals was evident, as the increased 8-OHDPAT-stimulated $\left[{ }^{35} \mathrm{~S}\right] \mathrm{GTP} \gamma \mathrm{S}$ binding [3] (Fig. 5, control $v s$ naïve). Neither the IL1-Ra nor the fluoxetine monotherapy significantly reduced the function of 5-HT1A autoreceptors (a small reduction in binding was only observed in IL-1ra-treated rats in response to $1 \mu \mathrm{M}$ of 8-OH-DPAT). The combined administration of IL-1ra and fluoxetine not only returned $\left[{ }^{35} \mathrm{~S}\right] \mathrm{GTP} \gamma \mathrm{S}$ binding to a normal level, but also suppressed 5-HT1A receptor function below the 1 observed in naïve animals (Fig. 5, IL-1ra + FLX vs naïve).

\section{Spontaneous Seizures}

Neither IL-1ra nor fluoxetine, nor their combination modified the frequency of behavioral stage 4 to 5 spontaneous seizures in post-SE rats (Fig. 6).

\section{Effects Treatments in Naïve Rats}

IL1-ra administration had no effects in behavioral, endocrine, biochemical, and autoradiography assays in naïve rats (Fig. 7). Treatment of naïve animals with fluoxetine did not affect their behavior, HPA axis response, and 5HT release; however, fluoxetine significantly suppressed the function of presynaptic 5-HT1A receptors (Fig. 7). No interaction between IL-1ra and fluoxetine was observed when
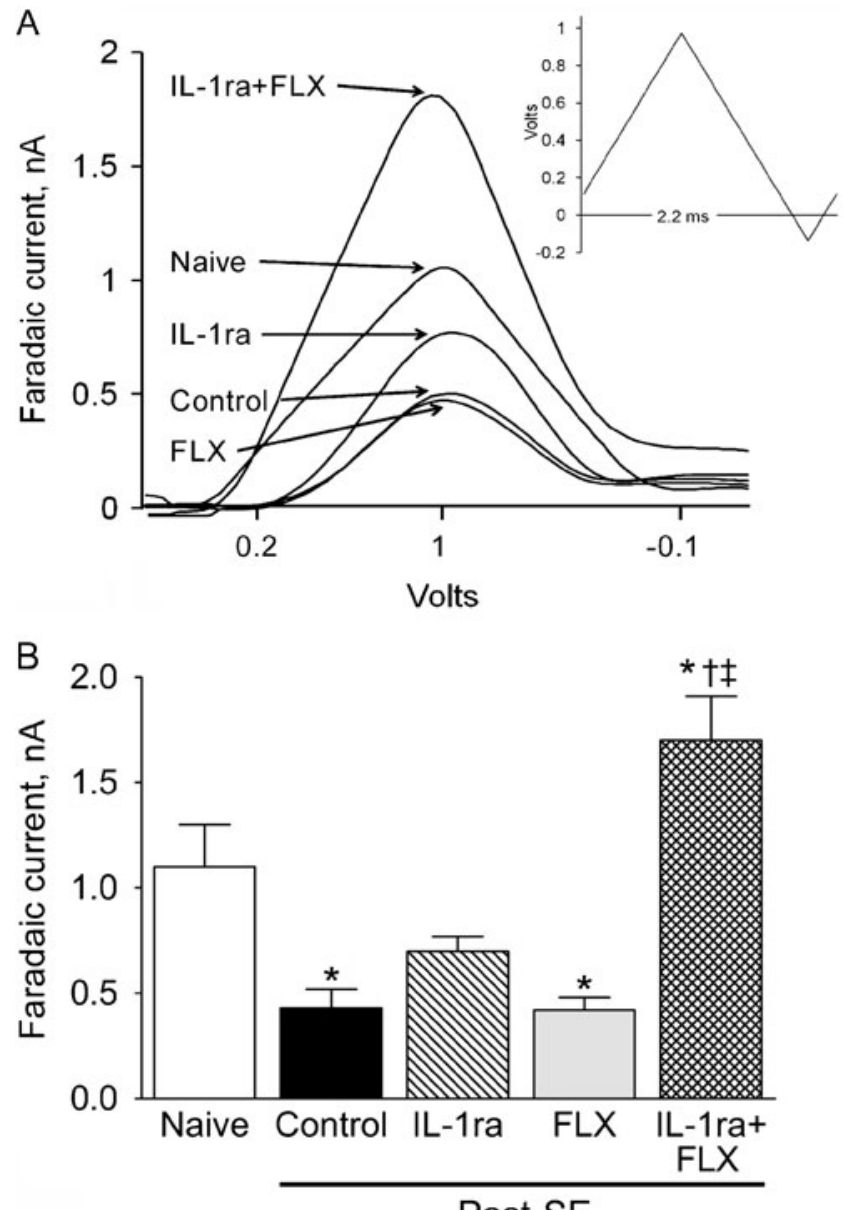

Post-SE

Fig. 4 Effects of interleukin-1 receptor antagonist (IL-1ra), fluoxetine (FLX), and their combination on the evoked 5-HT release from the cortex. (a) Sample voltagrams show the reduced 5-HT release in control (i.e., post-status epilepticus [SE], vehicle treated) as compared with naive rats; the lack of effects of fluoxetine in post-SE rats; the increased 5-HT release upon the IL1-ra administration; and the increase of 5-HT release greater than normal level after the combined IL-1ra/FLX treatment (compare with naïve animals). Inset shows the ramp current applied to dorsal raphe to evoke 5-HT release from the cortex. (b) Statistical analysis of the fast cyclic voltammetry assay. Each group included 6 rats. Data are shown as mean \pm SEM. ${ }^{*} p<0.05$ versus naïve; ${ }^{\dagger} p<0.05$ versus SE alone; ${ }^{\star} p<0.05$ versus IL-1ra alone and versus FLX alone (one-way analysis of variance + Neuman-Keuls post hoc test)

the drugs were administered together in any of the assays (not shown).

\section{Discussion}

The main findings of the present study are: i) IL-1ra suppresses the function of presynaptic 5-HT1A receptors in epileptic rats, and ii) the combined treatment with IL-1ra (which is partially effective when administered alone) and fluoxetine (which is ineffective when used as the monotherapy) leads to the complete reversal of all depression-like abnormalities, 


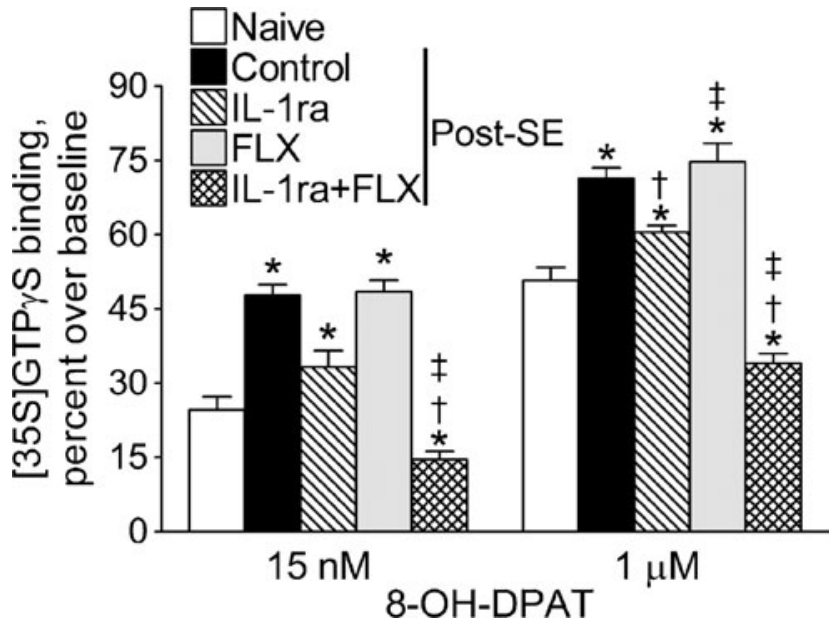

Fig. 5 Effects of interleukin-1 receptor antagonist (IL-1ra), fluoxetine (FLX), and their combination on the 8-hydroxy-2-dipropylaminotetralin (8-OH-DPAT)-stimulated $\left[{ }^{35} \mathrm{~S}\right] \mathrm{GTP} \gamma \mathrm{S}$ binding in dorsal raphe. Each group included 5 animals. Data are shown as mean \pm SEM. $* p<0.05$ versus naïve; ${ }^{\dagger} p<0.05$ versus status epilepticus (SE) alone; ${ }^{*} p<0.05$ versus IL-1ra alone and versus FLX alone (two-way analysis of variance + Bonferroni post-hoc test)

with the exception of the hyperactivity of the HPA axis, the latter remaining only partially improved. The findings are summarized in Table 1.

\section{Fluoxetine Monotherapy}

The study has confirmed our earlier data that epilepsyassociated depression-like state is resistant to fluoxetine. We have used several fluoxetine regimens, both reported [2] and unpublished, with doses ranging from 10 to $20 \mathrm{mg} / \mathrm{kg}$, treatment duration from 1 to 2 weeks, and injection frequency once and twice a day. None of the used protocols resulted in the alleviation of depressive impairments.

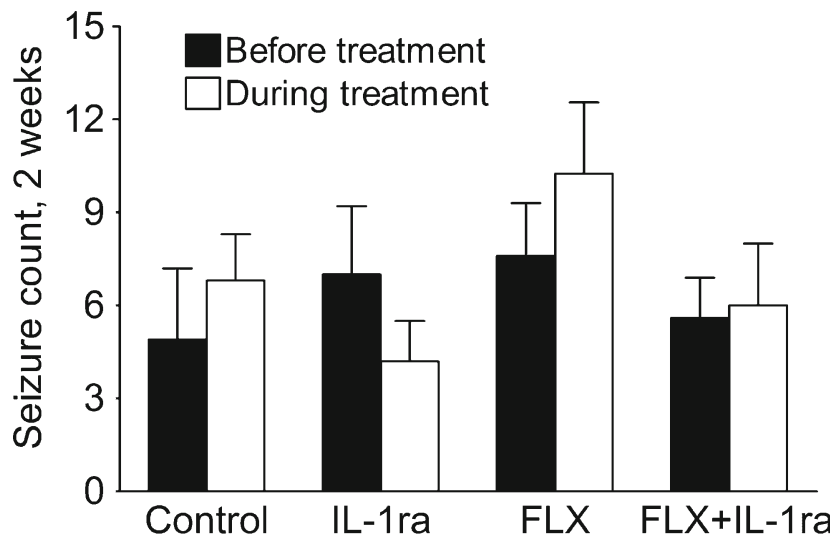

Fig. 6 Neither interleukin-1 receptor antagonist (IL-1ra) nor fluoxetine (FLX), nor their combination modified the frequency of behavioral spontaneous seizures. Data are shown as mean \pm SEM. Sample sizes: control, $\mathrm{n}=9$; IL1-ra, $\mathrm{n}=7$; FLX, $\mathrm{n}=7$; IL-1ra + FLX, $\mathrm{n}=8$. (Kruskal-Wallis test)
In addition to the already established inability of fluoxetine to improve behavioral and biochemical hallmarks of epilepsyassociated depression [2], we show here that the drug failed to normalize the HPA axis response, as well as the function of presynaptic 5-HT1 A receptors. Importantly, in naïve animals, fluoxetine did suppress the function of raphe 5-HT1A receptors, thus confirming that the unresponsiveness of post-SE rats specifically pertained to perturbations triggered by the epileptic process. The fact that fluoxetine-induced downregulation of presynaptic 5-HT1A receptors in naïve rats led to neither the increase of 5-HT release, nor to changes in behavior, reflects an apparent contribution of factors other than 5-HT1A-driven auto-inhibitory loop in the regulation of 5-HT release [31].

As mentioned in the "Introduction," the unresponsiveness to SSRI represents a significant problem in major depression [24, 25]. One of the widely discussed causes is a polymorphism of 5-HT1A receptor-encoding gene, Htr1a [32, 33]. A mechanism by which SSRI exert their antidepressant effects involves the desensitization of presynaptic 5-HT1A receptors due to the rise of the extracellular 5-HT [34-37]. It has been suggested that the ability of SSRI to desensitize presynaptic 5-HT1A receptors requires certain maximal level of their expression, and that individuals with the Htrla C(-1019)G polymorphism (referred to as $\mathrm{G} / \mathrm{G}$ allele carriers), are resistant to SSRI due to the previously described normal levels of these receptors $[32,38]$. At the same time, several studies failed to prove the association between this genetic trait and failure of SSRI to improve depression [39]. It would be reasonable to assume that the resistance to SSRI may stem from various causes, genetic and acquired alike.

\section{IL-1Ra Monotherapy}

The role of both brain and peripheral inflammation as a factor contributing to depression has been supported by clinical and experimental evidence, such as the presence of both brain and peripheral markers of inflammation in major depression patients; high frequency of comorbidity between primary autoimmune disorders and mood impairments; antidepressant effectiveness of anti-inflammatory drugs; the occurrence of clinical symptoms of depression in patients undergoing chemokine immunotherapy; the evolvement of behavioral and neuroendocrine depressive impairments in experimental animals treated with proinflammatory compounds [17, 40, 41]. Among various factors of inflammation, IL-1 $\beta$ appears to have particularly strong association with depression [17, 18, 20, 42]. Recent studies have reported the association between the polymorphism of IL1- $\beta$ promoter and major depression [43], and they have even implicated IL- $\beta$ gene polymorphism (specifically its rs16944 variant, which results in the increased IL1- $\beta$ levels) in the lack of response to SSRI [44].

IL- $\beta$ is an established modulator of function of the HPA axis $[18,19]$. For example, intrahippocampal administration 


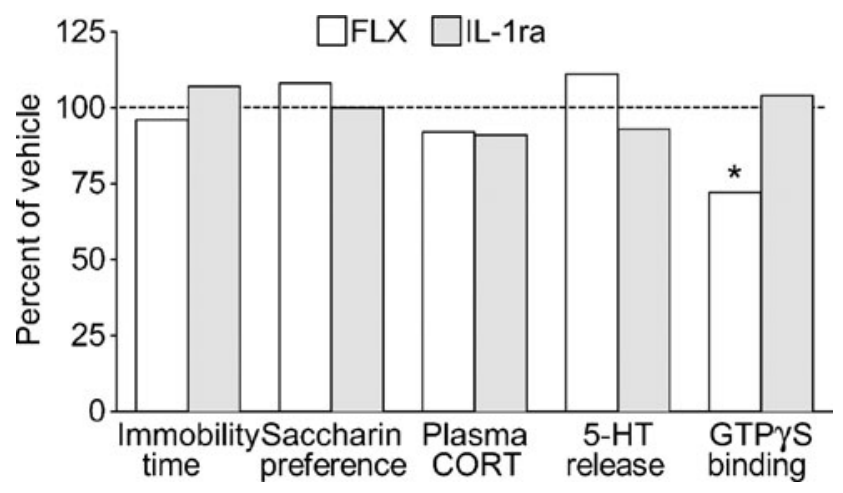

Fig. 7 Effects of interleukin-1 receptor antagonist (IL-1ra) and fluoxetine (FLX) in naïve rats. Each group included 4 animals. Data are presented as percent of vehicle-treated naïve animals. Neither of the treatments affected animals' performance in behavioral tests, plasma corticosterone (CORT) levels in response to $\mathrm{CRH}$ administration, and the evoked 5-HT release. Treatment with fluoxetine suppressed function of presynaptic 5-HT1A receptors. ${ }^{*} p<0.05$ versus control (Kruskal-Wallis + Mann-Whitney U post hoc test)

of recombinant IL1- $\beta$ in rats leads to the increased plasma CORT level [20]. This observation parallels events in epileptic rats, in which the increased expression of endogenous IL1- $\beta$ and its receptor [15] results in the hyperactivity of the HPA axis [7].

In turn, the dysregulation of the HPA axis has been regarded as a neuroendocrine hallmark of chronic stress [45, 46] and (in a form of either DEX suppression or the combined $\mathrm{DEX} / \mathrm{CRH}$ tests) has been used as a diagnostic tool in depression [47]. Mechanisms by which the dysregulated HPA axis leads to depression are apparently multiple. Several studies have explored the interaction between the HPA axis and serotonergic transmission, whereby excessively high levels of circulating glucocorticoids may upregulate presynaptic 5HT1A receptors and suppress raphe neuronal firing [48, 49]. On this note, we previously reported that pharmacological uncoupling of the HPA axis from raphe improved biochemical and behavioral hallmarks of depression in epileptic rats [3].
Along the lines of the discussed role of IL1- $\beta$ in epilepsy and depression, we have established that intrahippocampal delivery of IL-1ra improves the dysregulation of the HPA axis, compromised serotonergic transmission and behavioral deficit in the FST, and completely reverses the loss of saccharin preference (i.e., behavioral equivalent of anhedonia) in post-SE rats [12]. In the present study, we further show that central IL-1ra administration downregulates presynaptic 5-HT1A receptors.

\section{Combined Treatment with IL1-ra and Fluoxetine}

Remarkably, the combination of IL-1 ra with fluoxetine completely reversed hopelessness/despair-like behavioral impairments examined in the FST. At the same time, the lack of IL-1ra/fluoxetine interaction on the level of the HPA axis (Table 1) is expected, because the latter is situated upstream from the presumed target for fluoxetine (the upregulated raphe 5-HT1A autoreceptors).

Somewhat unexpected findings came from autoradiography and biochemical assays. The combined treatment with IL-1 ra and fluoxetine downregulated 5-HT-1A autoreceptors below the levels observed in naïve rats. Consequently, the evoked release of 5-HT from raphe exceeded the 1 detected in naïve subjects. IL1- $\beta$ is capable of directly reducing the firing of raphe serotonergic neurons [50]. It is conceivable that partial downregulation of presynaptic 5-HT1A receptors and the increased 5-HT release observed in our studies after IL-1ra monotherapy stemmed not only from resetting the function of the HPA axis, but also from its direct effects on raphe nucleus. Therefore, the combination of IL-1ra with fluoxetine could have resulted in the oversuppression of 5-HT1A autoreceptors and thus in the amplified 5-HT release. However, the hyperactive serotonergic tone did not translate into the further reduction of the immobility time in the FST, apparently because the active swimming/immobility ratio is defined by a combination of environmental and intrinsic factors beside

Table 1 Summary of Effects of IL-1ra, Fluoxetine and Their Combination on Epilepsy-Associated Depression-Like Impairments

\begin{tabular}{|c|c|c|c|c|c|}
\hline \multirow{2}{*}{$\begin{array}{l}\text { Groups } \\
\text { Parameters }\end{array}$} & \multirow[t]{2}{*}{ Naive } & \multicolumn{4}{|l|}{ Post-SE } \\
\hline & & Control & IL-1ra & Fluoxetine & IL-1ra + fluoxetine \\
\hline Increased immobility time in the FST & - & +++ & ++ & +++ & - \\
\hline Lack of saccharin preference & - & +++ & - & +++ & - \\
\hline HPA axis function & + & +++ & ++ & +++ & ++ \\
\hline Serotonergic tone & +++ & + & ++ & + & ++++ \\
\hline Presynaptic 5-HT1A function & ++ & ++++ & +++ & ++++ & + \\
\hline
\end{tabular}

Behavioral, neuroendocrine, biochemical, and receptor impairments in post-SE animals without (control) and with treatments are compared to normal values observed in naïve animals

FST =forced swim test; HPA=hypothalamo-pituitary-adrenocortical; IL-1ra=interleukin-1 receptor antagonist; SE=status epilepticus 
serotonergic transmission, and therefore cannot be modified beyond certain limits by modulating the 5 -HT release alone.

Our previous studies [12], as well as the present experiments, have shown that the loss of the taste preference in the saccharin intake test is highly responsive to IL-1 ra; indeed, unlike other depressive impairments, the loss of saccharin preference in post-SE animals was completely reversed by the IL-1ra monotherapy. Furthermore, our original report showed that anhedonia-like impairments in epileptic animals had no statistical association with the diminished 5-HT release from raphe nucleus [2]. It appears that the loss of taste preference in the pilocarpine model is driven primarily by the enhanced IL1$\beta$ signaling, and does not require serotonergic transmission.

Similar to our earlier report [12], antidepressant effects of IL-1a, as well as IL-1ra/fluoxetine combination were not associated with the modulation of spontaneous seizures.

Based on the reported data, we suggest that epilepsyinduced activation of the brain IL1- $\beta$ signaling triggers the cascade of the following events: the dysregulation of the HPA axis $\rightarrow$ upregulation of 5-HT1A autoreceptors $\rightarrow$ diminished serotonergic tone $\rightarrow$ behavioral symptoms of hopelessness/ despair. Anhedonia-like impairment primarily depends on the pathologically enhanced IL1- $\beta$ signaling, but not on perturbations in serotonergic transmission. Furthermore, the IL-1 $\beta$ driven upregulation of 5-HT1A autoreceptors renders fluoxetine ineffective. Consequently, the disruption of the IL1- $\beta$ signaling both mitigates symptoms of depression and restores therapeutic response to SSRI. Therefore, we propose that in certain forms of depression, including but not limited to depression associated with epilepsy, the resistance to SSRI may be driven by the pathologically enhanced IL1- $\beta$ signaling and subsequent upregulation of presynaptic 5-HT1A receptors. In such forms of depression, the use of IL1- $\beta$ blockers in conjunction with SSRI may represent an effective therapeutic approach.

Acknowledgments The study was supported by the National Institutes of Health research grants (R01 NS065783 and R21 MH079933, both to A.M.M.) and grant (R01 MH 52369 to J.G.H.). All authors have activities or relationships that can be perceived as conflicts of interests. Full conflict of interest disclosures are available in the electronic supplementary material for this article.

Required Author Forms Disclosure forms provided by the authors are available with the online version of this article.

\section{References}

1. Pineda E, Shin D, Sankar R, Mazarati AM. Comorbidity between epilepsy and depression: experimental evidence for the involvement of serotonergic, glucocorticoid, and neuroinflammatory mechanisms. Epilepsia 2010;51(Suppl 3):110-114.

2. Mazarati A, Siddarth P, Baldwin RA, Shin D, Caplan R, Sankar R. Depression after status epilepticus: behavioural and biochemical deficits and effects of fluoxetine. Brain 2008;131:2071-2083.
3. Pineda EA, Hensler JG, Sankar R, Shin D, Burke TF, Mazarati AM. Plasticity of presynaptic and postsynaptic serotonin 1 A receptors in an animal model of epilepsy-associated depression. Neuropsychopharmacology 2011;36:1305-1316.

4. Aghajanian GK, Sprouse JS, Sheldon P, Rasmussen K. Electrophysiology of the central serotonin system: receptor subtypes and transducer mechanisms. Ann N Y Acad Sci 1990;600:93-103.

5. Riad M, Garcia S, Watkins KC, et al. Somatodendritic localization of 5-HT1 A and preterminal axonal localization of 5-HT1B serotonin receptors in adult rat brain. J Comp Neurol 2000;417:181-194.

6. Sprouse JS, Aghajanian GK. Electrophysiological responses of serotoninergic dorsal raphe neurons to 5-HT1A and 5-HT1B agonists. Synapse 1987;1:3-9.

7. Mazarati AM, Shin D, Kwon YS, et al. Elevated plasma corticosterone level and depressive behavior in experimental temporal lobe epilepsy. Neurobiol Dis 2009;34:457-461.

8. Dinan T. Novel approaches to the treatment of depression by modulating the hypothalamic — pituitary — adrenal axis. Hum Psychopharmacol 2001;16:89-93.

9. Kanner AM. Depression and epilepsy: a review of multiple facets of their close relation. Neurol Clin 2009;27:865-880.

10. Zobel A, Wellmer J, Schulze-Rauschenbach S, et al. Impairment of inhibitory control of the hypothalamic pituitary adrenocortical system in epilepsy. Eur Arch Psychiatry Clin Neurosci 2004;254:303311.

11. Lothe A, Didelot A, Hammers A, et al. Comorbidity between temporal lobe epilepsy and depression: a [18F]MPPF PET study. Brain 2008;131:2765-2782.

12. Mazarati AM, Pineda E, Shin D, Tio D, Taylor AN, Sankar R. Comorbidity between epilepsy and depression: role of hippocampal interleukin-1beta. Neurobiol Dis 2010;37:461-467.

13. Mertens M, Singh JA. Anakinra for rheumatoid arthritis: a systematic review. J Rheumatol 2009;36:1118-1125.

14. Arend WP. Interleukin-1 receptor antagonist: discovery, structure and properties. Prog Growth Factor Res 1990;2:193-205.

15. Ravizza T, Gagliardi B, Noe F, Boer K, Aronica E, Vezzani A. Innate and adaptive immunity during epileptogenesis and spontaneous seizures: evidence from experimental models and human temporal lobe epilepsy. Neurobiol Dis 2008;29:142-160.

16. Vezzani A, French J, Bartfai T, Baram TZ. The role of inflammation in epilepsy. Nat Rev Neurol 2011;7:31-40.

17. Dantzer R. Cytokine, sickness behavior, and depression. Immunol Allergy Clin North Am 2009;29:247-264.

18. Goshen I, Yirmiya R. Interleukin-1 (IL-1): a central regulator of stress responses. Front Neuroendocrinol 2009;30:30-45.

19. Vezzani A, Maroso M, Balosso S, Sanchez MA, Bartfai T. IL-1 receptor/Toll-like receptor signaling in infection, inflammation, stress and neurodegeneration couples hyperexcitability and seizures. Brain Behav Immun 2011;25:1281-1289.

20. Parsadaniantz SM, Batsche E, Gegout-Pottie P, et al. Effects of continuous infusion of interleukin 1 beta on corticotropin-releasing hormone ( $\mathrm{CRH}), \mathrm{CRH}$ receptors, proopiomelanocortin gene expression and secretion of corticotropin, beta-endorphin and corticosterone. Neuroendocrinology 1997;65:53-63.

21. Jobe PC. Common pathogenic mechanisms between depression and epilepsy: an experimental perspective. Epilepsy Behav 2003;4: S14-S24.

22. Kondziella D, Alvestad S, Vaaler A, Sonnewald U. Which clinical and experimental data link temporal lobe epilepsy with depression? J Neurochem 2007;103:2136-2152.

23. Dantzer R, O'Connor JC, Lawson MA, Kelley KW. Inflammationassociated depression: from serotonin to kynurenine. Psychoneuroendocrinology 2011;36:426-436.

24. Barbui C, Hotopf M, Garattini S. Fluoxetine dose and outcome in antidepressant drug trials. Eur J Clin Pharmacol 2002;58:379386. 
25. Rush AJ, Trivedi MH, Wisniewski SR, et al. Acute and longer-term outcomes in depressed outpatients requiring one or several treatment steps: a STAR*D report. Am J Psychiatry 2006;163:1905-1917.

26. Hovorka J, Herman E, Nemcova II. Treatment of interictal depression with citalopram in patients with epilepsy. Epilepsy Behav 2000;1:444-447.

27. Kanner AM, Kozak AM, Frey M. The use of sertraline in patients with epilepsy: is it safe? Epilepsy Behav 2000;1:100-105.

28. Blumer D. Antidepressant and double antidepressant treatment for the affective disorder of epilepsy. J Clin Psychiatry 1997;58:3-11.

29. Wrona MZ, Dryhurst G. Oxidation chemistry of 5-hydroxytryptamine. 1. Mechanism and products formed at micromolar concentrations. J Org Chem 1987;52:2817-2825.

30. Hensler J, Durgam H. Regulation of 5-HT(1A) receptor-stimulated [35S]-GtpgammaS binding as measured by quantitative autoradiography following chronic agonist administration. Br J Pharmacol 2001;132:605-611.

31. Hokfelt T, Xu ZQ, Shi TJ, Holmberg K, Zhang X. Galanin in ascending systems: focus on coexistence with 5-hydroxytryptamine and noradrenaline. Ann N Y Acad Sci 1998;863:252-263.

32. Richardson-Jones JW, Craige CP, Guiard BP, et al. 5-HT1A autoreceptor levels determine vulnerability to stress and response to antidepressants. Neuron 2010;65:40-52.

33. Kato M, Fukuda T, Wakeno M, et al. Effect of 5-HT1A gene polymorphisms on antidepressant response in major depressive disorder. Am J Med Genet B Neuropsychiatr Genet 2009;150B:115123.

34. Chaput Y, de Montigny C, Blier P. Effects of a selective 5-HT reuptake blocker, citalopram, on the sensitivity of 5-HT autoreceptors: electrophysiological studies in the rat brain. Naunyn Schmiedebergs Arch Pharmacol 1986;333:342-348.

35. Le Poul E, Laaris N, Doucet E, Laporte AM, Hamon M, Lanfumey L. Early desensitization of somato-dendritic 5-HT1A autoreceptors in rats treated with fluoxetine or paroxetine. Naunyn Schmiedebergs Arch Pharmacol 1995;352:141-148.

36. Maudhuit C, Prevot E, Dangoumau L, Martin P, Hamon M, Adrien J. Antidepressant treatment in helpless rats: effect on the electrophysiological activity of raphe dorsalis serotonergic neurons. Psychopharmacology (Berl) 1997;130:269-275.

37. Blier P, Pineyro G, el Mansari M, Bergeron R, de Montigny C. Role of somatodendritic 5-HT autoreceptors in modulating 5-HT neurotransmission. Ann NY Acad Sci 1998;861:204-216.
38. Lemonde S, Turecki G, Bakish D, et al. Impaired repression at a 5hydroxytryptamine $1 \mathrm{~A}$ receptor gene polymorphism associated with major depression and suicide. J Neurosci 2003;23:8788-8799.

39. Levin GM, Bowles TM, Ehret MJ, et al. Assessment of human serotonin 1A receptor polymorphisms and SSRI responsiveness. Mol Diagn Ther 2007;11:155-160.

40. Dantzer R, Kelley KW. Stress and immunity: an integrated view of relationships between the brain and the immune system. Life Sci 1989;44:1995-2008.

41. Miller AH, Maletic V, Raison CL. Inflammation and its discontents: the role of cytokines in the pathophysiology of major depression. Biol Psychiatry 2009;65:732-741.

42. Konsman JP, Veeneman J, Combe C, Poole S, Luheshi GN, Dantzer R. Central nervous action of interleukin-1 mediates activation of limbic structures and behavioural depression in response to peripheral administration of bacterial lipopolysaccharide. Eur J Neurosci 2008;28:2499-2510.

43. Borkowska P, Kucia K, Rzezniczek S, et al. Interleukin-1beta promoter $(-31 \mathrm{~T} / \mathrm{C}$ and $-511 \mathrm{C} / \mathrm{T})$ polymorphisms in major recurrent depression. J Mol Neurosci 2011;44:12-16.

44. Baune BT, Dannlowski U, Domschke K, et al. The interleukin 1 beta (IL1B) gene is associated with failure to achieve remission and impaired emotion processing in major depression. Biol Psychiatry 2010;67:543-549.

45. Herman JP, Cullinan WE. Neurocircuitry of stress: central control of the hypothalamo-pituitary-adrenocortical axis. Trends Neurosci 1997;20:78-84

46. Yu S, Holsboer F, Almeida OF. Neuronal actions of glucocorticoids: focus on depression. J Steroid Biochem Mol Biol 2008;108:300-309.

47. Watson S, Gallagher P, Smith MS, Ferrier IN, Young AH. The DEX/CRH test-is it better than the DST? Psychoneuroendocrinology 2006;31:889-894.

48. Bellido I, Hansson AC, Gomez-Luque AJ, Andbjer B, Agnati LF, Fuxe K. Corticosterone strongly increases the affinity of dorsal raphe 5-HT1A receptors. Neuroreport 2004;15:1457-1459.

49. Judge $\mathrm{SJ}$, Ingram $\mathrm{CD}$, Gartside SE. Moderate differences in circulating corticosterone alter receptor-mediated regulation of 5-hydroxytryptamine neuronal activity. J Psychopharmacol 2004; 18:475-483.

50. Brambilla D, Franciosi S, Opp MR, Imeri L. Interleukin-1 inhibits firing of serotonergic neurons in the dorsal raphe nucleus and enhances GABAergic inhibitory post-synaptic potentials. Eur J Neurosci 2007;26:1862-1869. 